\title{
El uso del lisímetro de pesada en clavel (Dianthus caryophyllus L.) genera ahorro en fertirriego
}

\section{The use of the weighing lysimeter in carnation (Dianthus caryophyllus L.) generates saving in fertigation}

\author{
Germán A. Moreno-Poveda ; Víctor J. Flórez-Roncancio² ; Carlos A. González-Murillo³ \\ ${ }^{1}$ Ing. Agrónomo, M.Sc. Universidad Nacional de Colombia, Facultad de Ciencias Agrarias. Bogotá, D.C., Colombia; e-mail: gamorenop@ \\ unal.edu.co; iD https://orcid.org/0000-0002-6644-3213 \\ ${ }^{2}$ Ing. Agrónomo, M.Sc., Ph.D. Universidad Nacional de Colombia, Facultad de Ciencias Agrarias, Departamento de Agronomía. Bogotá, \\ D.C., Colombia; e-mail: vj lorezr@unal.edu.co; iDhttps://orcid.org/0000-0002-3081-2400 \\ ${ }^{3}$ Ing. Agrónomo. M.Sc. Universidad Nacional de Colombia, Facultad de Ingeniería, Departamento de Ingeniería Civil y Agrícola. Bogotá, \\ D.C., Colombia; e-mail: cagonzalezmu@unal.edu.co; iD https://orcid.org/0000-0002-2920-8425
}

Cómo citar: Moreno-Poveda, G.A.; Flórez-Roncancio, V.J.; González-Murillo, C.A. 2021. El uso del lisímetro de pesada en clavel (Dianthus caryophyllus L.) genera ahorro en fertirriego Rev. U.D.C.A Act. \& Div. Cient. 24(2):e1940. http://doi.org/10.31910/rudca.v24.n2.2021.1940

Artículo de acceso abierto publicado por Revista U.D.C.A Actualidad \& Divulgación Científica, bajo una Licencia Creative Commons CC BY-NC 4.0

Publicación oficial de la Universidad de Ciencias Aplicadas y Ambientales U.D.C.A, Institución de Educación Superior Acreditada de Alta Calidad por el Ministerio de Educación Nacional.

Recibido: abril 19 de 2021

Aceptado: septiembre 10 de 2021

Editado por: Helber Adrián Arévalo Maldonado

\section{RESUMEN}

El exceso o déficit en el volumen de fertirriego genera desbalances nutricionales, en razón a que influyen en el pH y la CE, afectando el desempeño de las plantas. Además, el descontrol del volumen de fertirriego puede causar excesos de lixiviados con efectos perjudiciales en el medioambiente. La fertirrigación en la producción de clavel en la Sabana de Bogotá, se hace de forma empiríca, por tanto, es indispensable ajustar el volumen de fertirriego a las necesidades del cultivo, para evitar los problemas mencionados. Por esta razón, se evaluó el efecto del control del volumen de fertirriego con lisímetro de peasada, en comparación con el manejo del fertirriego tradicional, en el desempeño de las plantas de miniclavel, sembradas en sutrato, en condiciones de invernadero. Para la evaluación, se realizó un diseño experimental en bloques completos al azar, con los tratamientos de sistema de cultivo con lisímetro (CL) y sistema de cultivo con fertirriego tradicional sin lisímetro (SL). Las variables evaluadas fueron volumen de riego, contenido nutricional de planta completa en cinco estadios fenológicos, $\mathrm{pH}$ y nutrientes mayores y secundarios $(\mathrm{Ca}-\mathrm{Mg})$, en el sustrato y en la solución drenada. El CL genera un ahorro en el volumen de fertirriego en comparación con el SL. Se encontraron diferencias en el continuo planta - sustrato - lixiviado, en $\mathrm{N}, \mathrm{K}, \mathrm{Mg}$, así como en el $\mathrm{pH}$ del sustrato. El CL fue más eficiente, al generar mayor acumulación de masa seca, con el uso de menos agua y nutrientes, sin afectar el rendimiento, ni la calidad.

Palabras clave: Cultivo sin suelo; Nutrientes; pH; Masa seca; Lisimetría. 


\section{ABSTRACT}

The amount of fertigation excess or deficit generates nutritional unbalances given it affects $\mathrm{pH}, \mathrm{CE}$, and the nutrients balance influencing the plant development performance. In addition, the uncontrolled fertigation volume can cause an excess of leachates with detrimental effects on the environment. The Fertigation in carnation production in the Bogotá savanna is done empirically, therefore, it is essential to adjust the fertigation volume to the needs of the crop to avoid the mentioned problems. For this reason, the effect of the control of the fertigation volume with weighting lysimeters was evaluated in comparison with the management of traditional fertigation in the performance of the spray carnation plants sown in substrate under greenhouse conditions. For the evaluation, an experimental design was carried out in complete randomized blocks with a lysimeter crop system (LCS) and a traditional crop system without a lysimeter (WLS) as treatments. Irrigation volume, whole plant nutritional content in five phenological stages, $\mathrm{pH}$, primary and secondary $(\mathrm{Ca}-\mathrm{Mg}$ ) nutrients in the substrate, and drainage solution were the variables evaluated. The LCS generates saving in the use of fertigation compared with WLS. Differences were found in the continuum plant - substrate - leached in $\mathrm{N}, \mathrm{K}, \mathrm{Mg}$, as well as in the $\mathrm{pH}$ of the substrate. The LCS treatment was more efficient by generating greater dry mass accumulation, with the use of less water and nutrients without affecting the yield or quality.

Keywords: Soilless culture system; Nutrients; pH; Dry mass; Llysimetry.

\section{INTRODUCCIÓN}

En Colombia, las plantas de clavel (Dianthus caryophyllus L.), se siembran en contenedores con sustrato; el aporte de los nutrientes, se realiza por concentración y el fertirriego por goteo, con bajos volúmenes en periodos cortos (2 a $8 \mathrm{~min}$ ) y altas frecuencias, buscando ofertar entre 1000 y $1700 \mathrm{~L} / \mathrm{m}^{2}$-año (Baracaldo et al. 2018; González-Murillo et al. 2021).

$\mathrm{El} \mathrm{pH}$ óptimo para cultivos de plantas ornamentales en medios sin suelo está entre 5,2 y 6,3; pH alcalinos generan problemas de absorción de $\mathrm{P}, \mathrm{Fe}, \mathrm{Mn}, \mathrm{B}, \mathrm{Zn}$ y $\mathrm{Cu}$ y los $\mathrm{pH}$ ácidos, problemas de absorción en N, K, Ca, Mg y Mo (Kafkafi \& Tarchitzky, 2011). El $\mathrm{pH}$ incrementa o disminuye principalmente, en razón a la diferencia entre la absorción de aniones y cationes, respectivamente: la primera está relacionada con la emisión de $\mathrm{HCO}_{3}$ u OH por parte de las plantas, mientras la absorción de cationes, se relaciona con la emisión de $\mathrm{H}_{3} \mathrm{O}$ (Sonneveld \& Voogt, 2009); cuando la absorción de $\mathrm{NH}_{4}{ }^{+}$excede la de $\mathrm{NO}_{3}{ }^{-}$, disminuye el $\mathrm{pH}$ de la solución y aumenta la absorción de la mayoría de los micronutrientes y al contrario el pH de la solución aumenta y disminuye la absorción de micronutrientes (Fageria, 2009). El curso del pH en el ambiente radical, sin suministro de $\mathrm{NH}_{4}{ }^{+}$en la solución nutriente, alcanzaría valores próximos a 7,0, mientras que con $15 \%$ de $\mathrm{N}_{-} \mathrm{NH}_{4}{ }^{+}$ disminuye por debajo de 5,0 (Sonneveld \& Voogt, 2009).
La disminución del pH también aumenta la disponibilidad de $\mathrm{P}$ y disminuye la absorción de cationes, como $\mathrm{Ca}, \mathrm{Mg}$ y $\mathrm{K}$ (Abasi et al. 2016) y, en consecuencia, serían fácilmente lixiviados. Con $\mathrm{pH}$ menor a 6, la mayoría del $\mathrm{Ca}$ adsorbido sigue siendo intercambiable, pero a medida que el $\mathrm{pH}$ aumenta, más cationes divalentes se adsorben específicamente. La absorción de cationes a $\mathrm{pH}$ bajo es menor que a mayores valores de $\mathrm{pH}$ y la absorción de $\mathrm{Ca}$, se deprime más que la absorción de otros cationes (Hawkesford et al. 2012).

La lisimetría caracteriza el balance hídrico generado entre la oferta de agua y los nutrientes a las plantas, la absorción de los mismos y el proceso de salida del sistema por lixiviación y evapotranspiración. El equipo provee una medida directa de la cantidad de agua usada en evapotranspiración por aislamiento y monitoreo continuo de un área de cultivo y como las mediciones se realizan en el sitio del cultivo, lo convierte en un método directo de medición (Rodríguez $\&$ Pire, 2008).

Por tanto, los lisímetros de pesada, generalmente, disponen de un sistema hidráulico o una celda de carga conectada a un sistema electrónico, que permiten obtener la pérdida de peso del contenedor, midiendo directamente la evapotranspiración del cultivo. Este es el caso del sistema UNLisitron, descrito en González Murillo et al. (2017) y de los lisímetros para estudios en algodón (Fisher, 2012), en tomate (Meijer et al. 1985) y en lechuga (Casanova P. et al. 2009). Aunque en los cultivos en sustrato se generan lixiviados entre el 20 y el 50 \% (Flórez R. et al. 2006) de la solución nutriente aplicada, en Colombia, no hay reportes del uso de lisímetros que propendan por el uso eficiente del agua, en el cultivo de clavel.

El objetivo de la investigación fue evaluar el control del volumen de fertirriego, monitoreado con lisímetro de pesada, en comparación con el manejo del fertirriego tradicional, sobre el desempeño de las plantas de clavel estándar.

\section{MATERIALES Y MÉTODOS}

El ensayo, se realizó en el Centro Agropecuario Marengo de la Universidad Nacional de Colombia, sede Bogotá, ubicado a una altitud de $2.543 \mathrm{~m}$ s.n.m., con promedios de temperatura y humedad relativa de $13,2{ }^{\circ} \mathrm{C}$ y $83 \%$, respectivamente; $4,6 \mathrm{~h}$ de brillo solar diario medio, $1,7 \mathrm{~m} \mathrm{~s}^{-1}$ de velocidad del viento y 690,6 mm de precipitación anual media (IDEAM, 2021).

Se dispuso de dos naves de un invernadero con estructura metálica y ventilación lateral y cenital pasiva, cada una de 6,8 x $50 \mathrm{~m}\left(340 \mathrm{~m}^{2}\right)$. Se construyeron 12 camas en madera $(15 \times 0,7 \mathrm{~m})$, a doble contenedor $(0,25 \mathrm{~m}$ de ancho $\mathrm{x} 0,15 \mathrm{~m}$ de profundidad cada uno) y levantadas a $0,4 \mathrm{~m}$ del suelo. Se sembraron esquejes con raíz de miniclavel cv. Rony, de color rojo, a una densidad de 24,7 plantas $/ \mathrm{m}^{2}$ de área de invernadero, en un sustrato compuesto de cascarilla de arroz tostada reutilizada, cascarilla de arroz tostada nueva y compost de residuos vegetales, en porcentajes de 60,30 y 10, respectivamente. 
Lisímetros y sistema de fertirriego. En los tratamientos con lisimetro, el lisimetro se instaló sobre una base metálica $(1 \times 0,3 \mathrm{~m})$. El equipo descrito en González Murillo et al. (2017), cuenta con un contenedor para las plantas y otro para la recolección del drenaje. Para el suministro de riego y fertirriego, se instaló un sistema de bombeo independiente para cada cama del experimento, operado con un programador automático de riego (controlador), tipo jardín.

Se utilizaron cuatro bombas de 1/2 HP: cada una de tres de ellas aportaba el riego de dos camas de cultivo, según la información obtenida del lisímetro de pesada y la cuarta bomba, el riego para las camas testigo. Por cama, se utilizaron dos líneas de goteo de 16 $\mathrm{mm}$, con goteros de tipo autocompensado y caudal de $1,1 \mathrm{~L} \mathrm{~h}^{-1}$, insertados en línea cada $15 \mathrm{~cm}$. El volumen de fertirriego, se ajustó al desarrollo del cultivo, en semanas después de la siembra (SDS): entre 3 y 14 SDS, el promedio suministrado fue de 60L/cama/día; de 15 a 26 SDS, se incrementó a 80L/cama/día y se finalizó con un promedio de 50L/cama/día.

Durante las dos primeras semanas, después de la siembra, se regó con agua y, en seguida, hasta la $16 \mathrm{SDS}$, se aplicó la fórmula de fertirriego para la fase vegetativa, con (mg.L $\left.{ }^{-1}\right)$ : $\mathrm{N}(220), \mathrm{P}(30)$, $\mathrm{K}(130), \mathrm{Ca}(120), \mathrm{Mg}(40), \mathrm{Fe}(3), \mathrm{Zn}(0,5), \mathrm{Cu}(1), \mathrm{B}(2)$ у $\mathrm{Mo}(0,1)$; luego, manteniendo $\mathrm{Ca}, \mathrm{Mg}, \mathrm{Fe}, \mathrm{Cu}$ y $\mathrm{Mo}$, se cambió en la fórmula para la fase productiva a N(165), P(25), K(190), Zn(1) y B(1,5).

Variables evaluadas. En las semanas 0 (semana de siembra), 7 SDS (dos semanas después de pinch), 14 SDS (transición de vegetativo a reproductivo), 21 SDS (pico de desbotone) y 28 SDS (punto de corte), se realizaron pesajes de la masa seca y análisis de tejido en planta completa, así como caracterización química del sustrato y de la solución drenada, para un total de 30 muestreos durante el ciclo (5 muestreos $* 2$ tratamientos $* 3$ repeticiones).
El cálculo de elementos mayores y secundarios absorbidos por hectárea de cultivo, a primer pico de cosecha, se hizo con base en el análisis del tejido vegetal. Para ello, se utilizó el modelo citado por Bertsch (2003):

$$
\frac{\mathrm{kg} N u t}{H a}=\frac{\operatorname{PS}\left(\frac{\mathrm{kg}}{H_{a}}\right) * \text { Nut } \%}{100}
$$

Ecuación 1

Donde: Nut $=$ nutriente PS $=$ peso seco Nut $\%=$ porcentaje de nutriente presente en el tejido vegetal.

Se utilizó un diseño experimental en bloques completos al azar, con dos tratamientos (cama sin lisímetro - testigo comercial y cama con lisímetro) y tres repeticiones, considerando una cama como repetición. Con los resultados de las variables medidas, se realizaron pruebas de verificación de supuestos para análisis paramétricos y análisis de varianza, con el uso del software estadístico SAS 9,0. Para las variables que mostraron evidencia de diferencias significativas, se aplicó la prueba de Tukey, al $5 \%$ de probabilidad.

\section{RESULTADOS Y DISCUSIÓN}

Considerando la dinámica del proceso de ahorro en fertirriego, ilustrada en la tabla 1 , se presentan los resultados sobre nutrientes en tejido, en sustrato y en solución lixiviada, obtenidos en el sistema de cultivo con lisímetro (CL) y sin lisímetro (SL) de pesada. En el tratamiento CL, se obtuvo un ahorro promedio de agua y nutrientes del 13,2\%, para las 28 semanas de ciclo productivo; asimismo, como los drenajes respectivos para los manejos SL y CL, fueron de 36,6 y $26,8 \%$, el aporte de lixiviados con potencial contaminante disminuyó en $9,8 \%$.

Tabla 1. Ahorro mensual de agua y de nutrientes durante un ciclo de cultivo, en el sistema de producción de miniclavel cv. Rony, con lisímetro de pesada, en comparación con el manejo del fertirriego, sin lisímetro de pesada.

\begin{tabular}{|c|c|c|c|c|c|c|c|}
\hline $\begin{array}{c}\text { Semanas } \\
\text { después de } \\
\text { siembra }\end{array}$ & 0 a 4 & 5 a 8 & 9 a 12 & 13 a 16 & 17 a 20 & 21 a 24 & 25 a 28 \\
\hline $\begin{array}{c}\text { Sin Lisímetro } \\
\left(\mathbf{L ~ m}^{-2}\right)\end{array}$ & 36,8 & 59,5 & 80,5 & 79,3 & 45,2 & 48,7 & 48,5 \\
\hline $\begin{array}{c}\text { Con Lisímetro } \\
\left(\mathbf{L ~ m}^{-2}\right)\end{array}$ & 63,0 & 54,8 & 55,5 & 57,8 & 40,1 & 42,9 & 47,1 \\
\hline $\begin{array}{c}\text { Ahorro de } \\
\text { agua } \\
(\%)\end{array}$ & 0 & 8 & 31 & 27 & 11 & 12 & 3 \\
\hline
\end{tabular}

\section{Caracterización química del tejido}

Masa seca. Se observó una tendencia de mayor acumulación de masa seca en las plantas con el fertirriego monitoreado CL, en comparación con el manejo del fertirriego tradicional, evidenciándose una diferencia aproximada de $1 \mathrm{~g}$ de la 21 a la 28
SDS. Al respecto el resultado del tratamiento SL concuerda con Kelso B. et al. (2012) quienes para clavel sembrado en sustrato muestran que, a la $25 \mathrm{SDS}$, la masa de la planta se encuentra entre 5 y 6 g. La diferencia en ganancia de masa seca entre los tratamientos se podría deber a la acidificación paulatina del sustrato en el tratamiento SL, lo que podría generar un efecto negativo 
sobre la fotosíntesis y menor acumulación de fotoasimilados. En condiciones de sobreoferta de agua, Dat et al. (2004) reportan cambios en el $\mathrm{pH}$ y en el potencial redox del suelo, debido a la dinámica de $\mathrm{H}^{+}$y el balance de cationes. El manejo tradicional del riego generaría estrés por el exceso de agua, condición que limita el crecimiento y la acumulación de masa seca (Li et al. 2004). Plantas de trigo crecidas sobre lisímetros presentaron mayor crecimiento y acumulación de masa seca, debido al aporte eficiente y preciso de agua y nutrientes, comparado con el manejo tradicional SL ( $\mathrm{Gu}$ et al. 2014).

Concentración y extracción de nutrientes. En la tabla 2 se observa que los valores para $\mathrm{N}, \mathrm{K}$ y $\mathrm{Mg}$ son significativamente mayores en las plantas creciendo en el tratamiento CL, en las semanas 28,
21-28 y 28, respectivamente, en comparación con el manejo del fertirriego tradicional. Al comparar $\mathrm{N}$ con el valor de $2,6 \%$ en tejido, reportado como óptimo, por Vélez-Carvajal et al. (2014) en clavel, estaría en deficiencia en algunos de los estadios evaluados. El P, con un promedio de $0,55 \%$, se presenta por encima del rango de 0,24 a 0,32 ; el ion $\mathrm{K}$, con un promedio de 3,19\%, se encontró cercano a $4 \%$, que es el valor óptimo; $\mathrm{Ca}$ y $\mathrm{Mg}$ con valores promedio de 1,28 y $0,43 \%$, respectivamente, en las cinco etapas evaluadas, se encuentran en un nivel normal.

Los valores de absorción para los elementos evaluados calculados a primer pico de producción de tallos florales (28 SDS), se presentan en la tabla 3. Las diferencias numéricas observadas entre los dos tratamientos, se relacionan directamente con la acumulación de

Tabla 2. Promedios de acumulación de masa seca y de concentración de nutrientes en planta completa de miniclavel cv. Rony, comparados entre las mismas semanas para cada tratamiento (manejos de fertirriego con y sin lisímetro de pesada).

\begin{tabular}{|c|c|c|c|c|c|c|c|}
\hline \multirow{2}{*}{ Tratamientos } & \multirow{2}{*}{ Semana } & \multirow{2}{*}{$\begin{array}{c}\text { Masa seca } \\
\text { (mg/planta) }\end{array}$} & $\mathbf{N}$ & $\mathbf{P}$ & $\mathbf{K}$ & $\mathrm{Ca}$ & $\mathbf{M g}$ \\
\hline & & & \multicolumn{5}{|c|}{$\%$} \\
\hline \multirow{5}{*}{ Con Lisímetro } & 0 & $35,7 \mathrm{a}$ & $2,20 \mathrm{a}$ & $0,35 \mathrm{a}$ & $1,55 \mathrm{a}$ & $1,13 \mathrm{a}$ & $0,44 a$ \\
\hline & 7 & $819,2 \mathrm{a}$ & $3,49 \mathrm{a}$ & $0,66 \mathrm{a}$ & $3,23 a$ & $1,75 \mathrm{a}$ & $0,51 \mathrm{a}$ \\
\hline & 14 & $3583,8 \mathrm{a}$ & $2,47 \mathrm{a}$ & $0,50 \mathrm{a}$ & $3,73 a$ & $1,43 a$ & $0,44 a$ \\
\hline & 21 & $6460,6 a$ & $2,91 \mathrm{a}$ & $0,60 \mathrm{a}$ & $3,72 \mathrm{a}$ & $1,06 \mathrm{a}$ & $0,43 a$ \\
\hline & 28 & $6479,9 \mathrm{a}$ & $2,03 a$ & $0,57 \mathrm{a}$ & $3,84 a$ & $1,28 \mathrm{a}$ & $0,40 \mathrm{a}$ \\
\hline \multirow{5}{*}{ Sin Lisímetro } & 0 & $29,5 \mathrm{a}$ & $2,21 \mathrm{a}$ & $0,35 \mathrm{a}$ & $1,55 a$ & $1,13 \mathrm{a}$ & $0,44 a$ \\
\hline & 7 & $745,6 a$ & $3,60 \mathrm{a}$ & $0,70 \mathrm{a}$ & $3,34 a$ & $1,58 \mathrm{a}$ & $0,51 \mathrm{a}$ \\
\hline & 14 & $3346,2 \mathrm{a}$ & $2,58 \mathrm{a}$ & $0,60 \mathrm{a}$ & $3,81 \mathrm{a}$ & $1,25 \mathrm{a}$ & $0,40 \mathrm{a}$ \\
\hline & 21 & $5425,5 \mathrm{a}$ & $2,60 \mathrm{a}$ & $0,58 \mathrm{a}$ & $3,45 b$ & $1,04 a$ & $0,39 \mathrm{a}$ \\
\hline & 28 & $5480,7 a$ & $1,82 \mathrm{~b}$ & $0,62 \mathrm{a}$ & $3,52 \mathrm{~b}$ & $1,23 \mathrm{a}$ & $0,34 \mathrm{~b}$ \\
\hline
\end{tabular}

Promedios seguidos por la misma letra entre tratamientos no presentan diferencias significativas, según la prueba de Tukey ( $\mathrm{p} \leq 0,05)$.

masa seca; en los dos tratamientos, se observa que la tendencia del $\mathrm{N}$ es creciente hasta la semana 21, etapa, en la cual, el botón floral se encuentra en pleno desarrollo. Navarro \& Navarro (2003) encontraron los mayores contenidos de $\mathrm{N}$ en los tejidos jóvenes, aunque con el desarrollo de la planta se eleva la relación C:N.
El P muestra, en los dos tratamientos, un comportamiento ascendente, lo que coincide con Kleiber \& Komosa (2008), para quienes el contenido de $\mathrm{P}$, en tejidos foliares de anturio, es ascendente, con relación de acumulación positiva en función del desarrollo vegetativo, cuando se presenta la mayor pendiente de acumulación en tejidos.

Tabla 3. Promedios de absorción de nutrientes en plantas de miniclavel cv. Rony, cultivadas en sustrato y sometidas al manejo del fertirriego, con apoyo de lisímetro de pesada y al manejo del fertirriego tradicional.

\begin{tabular}{|c|c|c|c|c|c|c|c|}
\hline \multirow{2}{*}{ Tratamiento } & \multirow{2}{*}{ Semana } & \multirow{2}{*}{$\begin{array}{c}\text { Masa Seca } \\
\left(\mathrm{kg} \mathrm{ha}^{-1}\right)\end{array}$} & $\mathbf{N}$ & $\mathbf{P}$ & $\mathbf{K}$ & $\mathrm{Ca}$ & $\mathbf{M g}$ \\
\hline & & & \multicolumn{5}{|c|}{$\left(\mathrm{kg} \mathrm{ha}^{-1}\right)$} \\
\hline \multirow{5}{*}{ Con Lisímetro } & 0 & 8,36 & 0,18 & 0,03 & 0,13 & 0,09 & 0,04 \\
\hline & 7 & 192,04 & 6,70 & 1,27 & 6,20 & 3,36 & 0,98 \\
\hline & 14 & 840,16 & 20,75 & 4,17 & 31,34 & 12,04 & 3,70 \\
\hline & 21 & 1514,57 & 44,07 & 9,09 & 56,34 & 15,45 & 6,51 \\
\hline & 28 & 1519,09 & 30,84 & 8,61 & 58,33 & 19,49 & 6,08 \\
\hline \multicolumn{2}{|l|}{ Total } & 4074,22 & 102,55 & 23,17 & 152,35 & 50,44 & 17,30 \\
\hline \multirow{5}{*}{$\begin{array}{c}\text { Sin } \\
\text { Lisímetro }\end{array}$} & 0 & 6,93 & 0,15 & 0,02 & 0,11 & 0,08 & 0,03 \\
\hline & 7 & 174,80 & 6,30 & 1,23 & 6,01 & 2,76 & 0,90 \\
\hline & 14 & 784,45 & 20,24 & 4,68 & 29,89 & 9,81 & 3,14 \\
\hline & 21 & 1271,91 & 33,11 & 7,38 & 43,88 & 13,19 & 4,96 \\
\hline & 28 & 1284,84 & 23,38 & 7,97 & 45,23 & 15,76 & 4,41 \\
\hline \multicolumn{2}{|l|}{ Total } & 3522,93 & 83,19 & 21,28 & 125,11 & 41,59 & 13,44 \\
\hline
\end{tabular}


El $\mathrm{K}$ tuvo su mayor incremento en la transición de crecimiento vegetativo a reproductivo, lo que coincide con Barrera-Aguilar et al. (2012), quienes en Lilium muestran un aumento en el requerimiento de $\mathrm{K}$, en la fase de crecimiento reproductivo, cuando hay exportación intensa hacia botones florales y flores en formación.

En ambos tratamientos, los elementos $\mathrm{Ca}$ y $\mathrm{Mg}$ muestran incrementos continuos en extracción, aunque para $\mathrm{Mg}$, se observa una ligera disminución, una vez diferenciada la flor y hasta primer pico de producción, lo cual, difiere de Vélez Carvajal et al. (2014), quienes en clavel observaron un comportamiento variado, en cuanto a la concentración del elemento en tejidos, durante el desarrollo del cultivo. Baracaldo et al. (2018) atribuyen la disminución de $\mathrm{Mg}$ al incremento de $\mathrm{NH}_{4}^{+}$en la fórmula de fertirriego que, a su vez, disminuyó el pH en el sustrato.

Comportamiento de $\mathrm{N}, \mathrm{P}, \mathrm{K}, \mathrm{Ca}$ y $\mathrm{Mg}$ en el sustrato y en la solución lixiviada. Los contenidos de $\mathrm{N}$ y $\mathrm{K}$ en el sustrato y en el lixiviado, así como el de $\mathrm{Mg}$ en el sustrato, a lo largo del ciclo de producción de los tallos florales, presentaron diferencias significativas (Figura 1). Para el tratamiento SL, se encontró que el contenido de $\mathrm{N}$, en la 21 SDS, era mayor en el lixiviado y en la 28 SDS, menor en el sustrato, lo que indicaría mayores pérdidas de dicho elemento.

El análisis de $\mathrm{K}$ en sustrato y lixiviado muestra una tendencia de mayores contenidos a partir de la 14 SDS en el tratamiento SL,
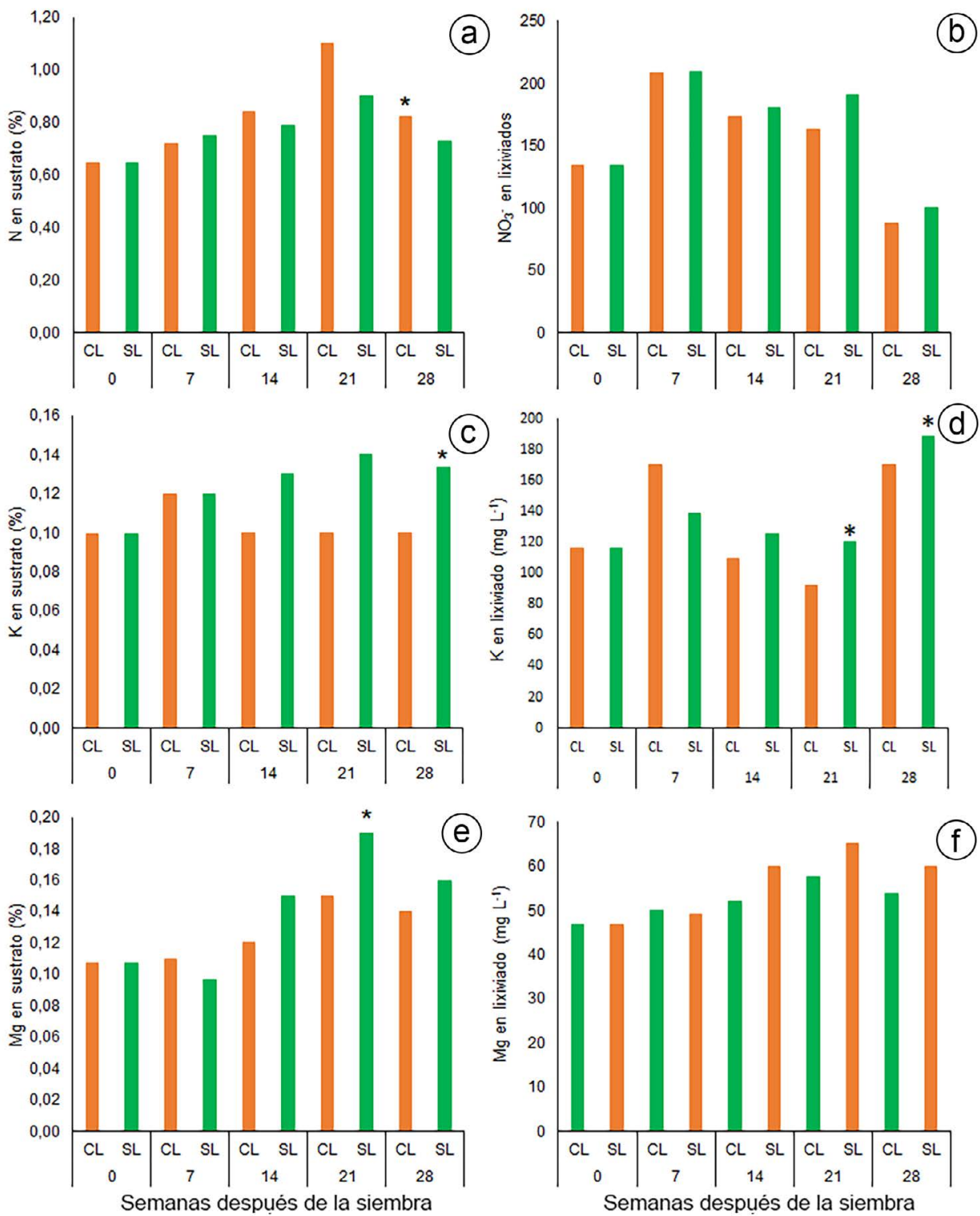

Figura 1. Comportamiento de nitrógeno $(\mathrm{N})$, potasio $(\mathrm{K})$ y magnesio $(\mathrm{mg})$ en sustrato y lixiviado, a lo largo de un pico de producción de tallos florales en plantas de miniclavel cv. Rony, cultivadas en sustratos y sometidas a manejo del fertirriego, con apoyo de lisímetro de pesada (CL) y al manejo del fertirriego tradicional (SL). Porcentajes en el sustrato de: a. nitrógeno; c. potasio; e. magnesio y contenidos en el lixiviado de: b. nitrato; d. potasio; f. magnesio.

$*$ Representa diferencias significativas según la prueba de Tukey $(\mathrm{p} \leq 0,05)$. 
en comparación con el manejo tradicional. Estas diferencias son significativamente mayores en el tratamiento SL, en la 28 SDS, para el contenido del ion en sustrato (Figura 1c) y en la 21 y 28 SDS, en lixiviado (Figura 1d); esto indicaría acumulación del nutriente en sustrato por dificultad en su absorción y mayores pérdidas por lixiviación de K. Para $\mathrm{N}$ y K las diferencias estadísticas están precedidas de tendencias numéricas, a partir de la 14 SDS, semana posterior al ahorro más representativo en el tratamiento con fertirriego monitoreado CL.

De forma independiente de los tratamientos, se destaca que la concentración de $\mathrm{NO}_{3}{ }_{3}^{-}$en los lixiviados alcanza un nivel máximo de $210 \mathrm{mg} \mathrm{L}^{-1}$, reafirmando el potencial contaminante de las fórmulas de fertirriego, utilizadas en la producción de clavel (Pacheco Avila et al. 2002).

A partir de la 14 SDS, también se advierte la tendencia de menor retención de $\mathrm{Mg}$, por parte del sustrato, en el tratamiento de manejo del fertirriego CL; en consecuencia, mayores pérdidas del nutriente en el lixiviado del tratamiento de fertirriego con manejo tradicional (Figuras 1e y f). Esta diferencia de menor retención de $\mathrm{Mg}$ por el sustrato, se hizo significativa en el tratamiento CL hacia la 21 SDS (Figura 1e).

Los nutrientes $\mathrm{P}$ y $\mathrm{Ca}$ no presentaron diferencias estadísticas entre los dos tratamientos en sustrato ni en lixiviado, aunque tendieron a acumularse en el sustrato, durante el desarrollo de las plantas. En el lixiviado, el P, a diferencia del $\mathrm{Ca}$, decrece a partir de la semana 14 SDS, lo que podría estar relacionado con un proceso de acumulación en tejido en constante crecimiento, pese al cambio de la cantidad aportada del nutriente en la fórmula de fertirriego, que disminuye su aporte en $20 \%$, a partir de la 14 SDS. De esta forma, se tendría una demanda constante por la planta, disminución del aporte en el fertirriego y menor pérdida en lixiviado.

El comportamiento de Ca en lixiviado fue más estable en el tiempo, lo que estaría relacionado con la estabilidad del nutriente en la fórmula de fertirriego, pese al cambio de desarrollo vegetativo a reproductivo (datos no mostrados).

$\mathrm{pH}$. En la figura 2, se muestra que la variable $\mathrm{pH}$ en el sustrato se mantuvo entre 6 y 7 , posiblemente, influenciado por la cascarilla de arroz que, según Arreaza (2000), tiende a generar un $\mathrm{pH}$ básico, al menos durante el primer año de uso. En la 28 SDS, se destaca el valor significantemente menor en el tratamiento de manejo tradicional del fertirriego, en comparación con el tratamiento CL.

Interacción entre variables. La discusión entre las variables, se plantea con base en: i) el sistema CL generó 13,2 \% menos aporte hídrico en el ciclo evaluado; ii) existe una diferencia numérica de $1 \mathrm{~g}$ en acumulación de masa seca, cuya tendencia se empieza a definir en la 14 SDS, cuando se obtuvo el ahorro más

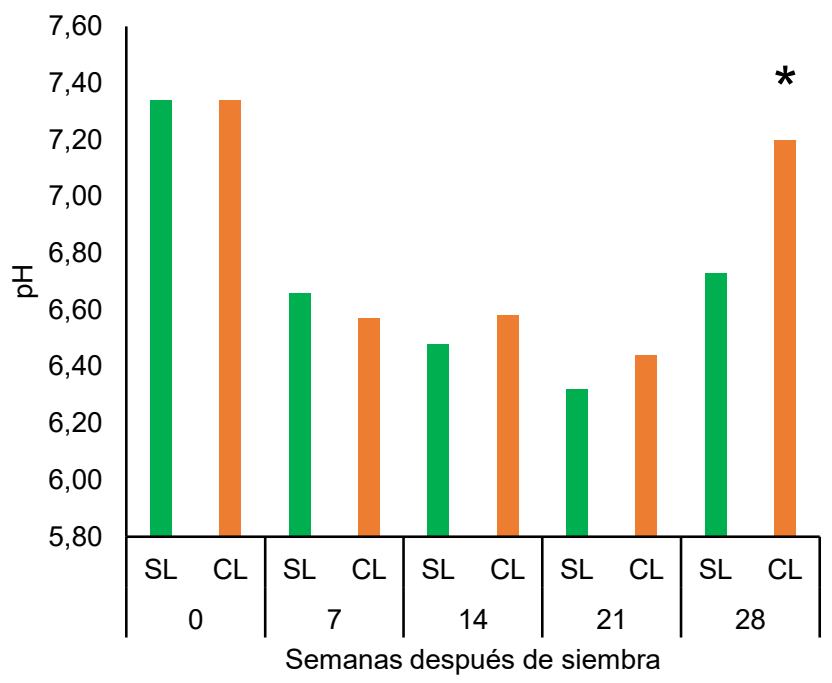

Figura 2. Comportamiento del $\mathrm{pH}$ en el sustrato del sistema productivo de miniclavel cv Rony, con manejo de fertirriego con apoyo de lisímetro (CL) y con manejo de fertirriego tradicional (SL).

*Representa diferencias significativas según la prueba de Tukey $(\mathrm{p} \leq 0,05)$.

representativo de agua en el sistema CL; iii) en los nutrientes $\mathrm{N}, \mathrm{K}$ y Mg, se reportaron diferencias estadísticas, en cuanto a la acumulación en tejido, lo cual, tiene una relación directa con el comportamiento de estos nutrientes en sustrato y en lixiviado y iv) en el sistema de manejo SL, se encontró una tendencia de acidificación del sustrato, al avanzar las semanas de cultivo.

En la figura 3a, se muestra la interacción entre ahorro de agua en el tratamiento CL y la tendencia de acumulación de masa seca. La diferencia en ganancia de masa seca entre los dos tratamientos estaría relacionada con sobreoferta de agua en el tratamiento SL. Dat et al. (2004) asocian esta sobreoferta con cambios en el $\mathrm{pH}$ y en el potencial redox del sustrato, debido a la dinámica de hidrogeniones y al balance de cationes, como también, con la solubilidad y la consecuente fitotoxicidad de metales pesados. 


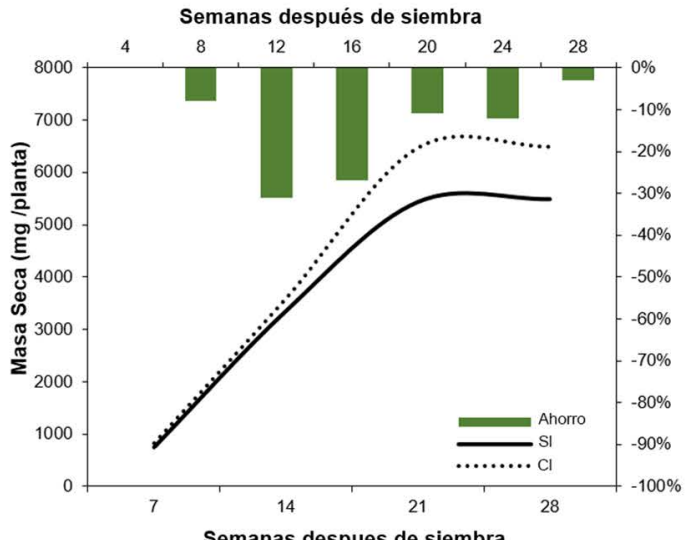

(a)
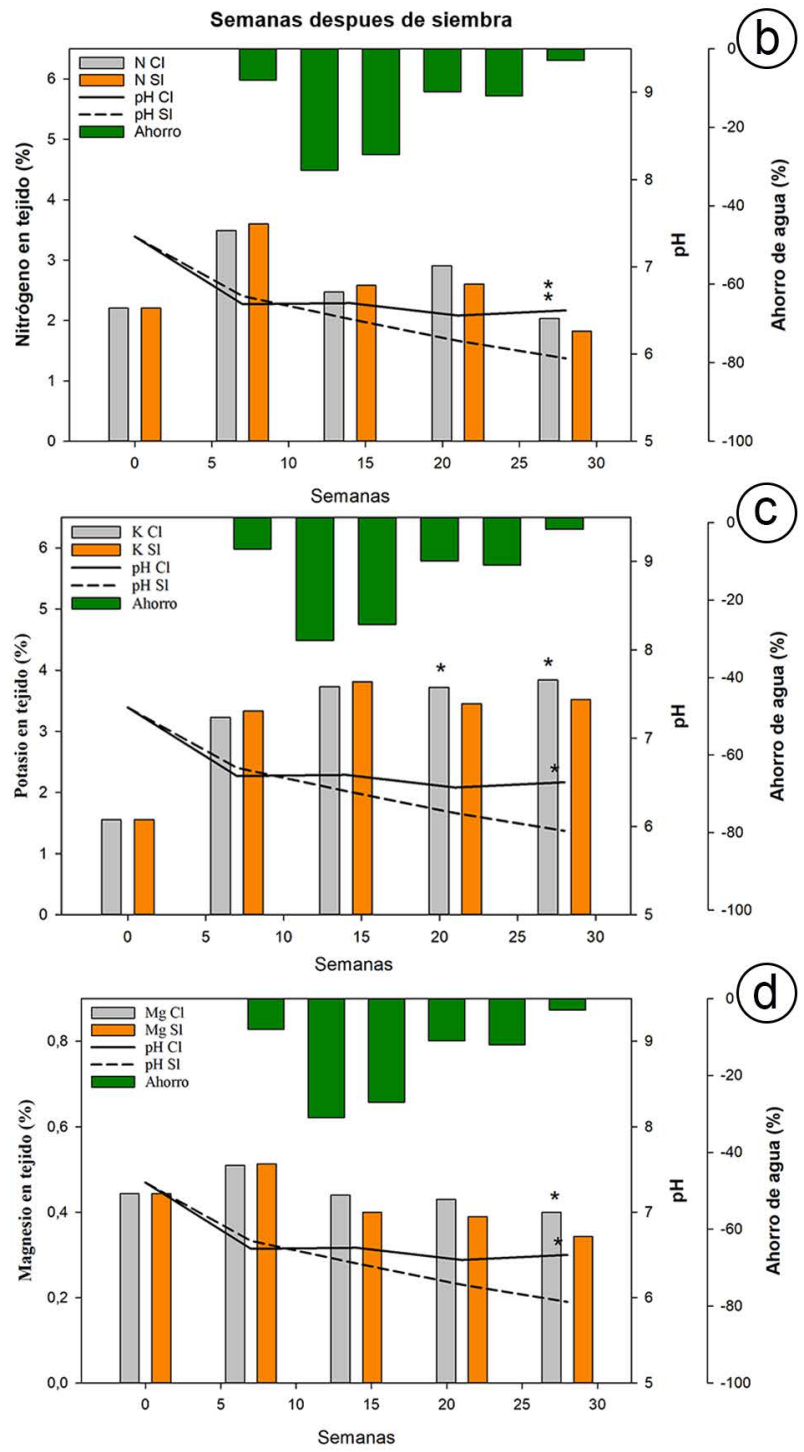

Figura 3. Sistema productivo de miniclavel cv Rony, con manejo de fertirriego con apoyo de lisímetro (CL) y con manejo de fertirriego tradicional (SL). a. acumulación de masa seca y ahorro de agua; b. comportamiento del parámetro pH en el sustrato; c. comportamiento de potasio en tejido (K), $\mathrm{pH}$ del sustrato y ahorro de agua; d. comportamiento de magnesio en tejido (Mg), $\mathrm{pH}$ del sustrato y ahorro de agua.

Por tanto, se plantea la interacción ahorro de fertirriego, $\mathrm{pH}$ en sustrato y dinámica de nutrientes en tejido, con énfasis en $\mathrm{N}, \mathrm{K}$ y $\mathrm{Mg}$, por ser los que presentaron diferencias significativas entre tratamientos. Al comparar los dos tratamientos, en la figura $3 \mathrm{~b}$, se aprecia el cambio en la tendencia del $\mathrm{pH}$, generándose acidificación en el tratamiento con manejo convencional, lo que se acentúa en la medida que se aporta más agua al sistema, confirmando lo expuesto por Dat et al. (2004). A medida que se acidifica el pH en 
el sustrato del tratamiento SL, se acumula menos $\mathrm{N}$ en el tejido, en comparación con el tratamiento CL, alcanzando diferencia significativa en la 28 SDS. Esto se relaciona con menos cantidad de $\mathrm{N}$ en el sustrato y mayor contenido de NO3- en el lixiviado (Figuras 1a y b), diferencias que se hacen significativas en las 28 y 21 SDS, respectivamente. Esta dinámica concuerda con lo expuesto por Sonneveld \& Voogt (2009), en cuanto que la absorción de cationes excede la de aniones y el $\mathrm{pH}$ en la rizosfera, decrece.

Teniendo en cuenta que el manejo de $\mathrm{N}$ se realizó en una relación nítrico: amoniacal 80:20 y de acuerdo con Bloom et al. (1992) y Carpenter et al. (1998), el N-NO3- es soluble, móvil en el suelo y no retenido por las cargas negativas de los coloides, es posible inferir que el exceso de agua en el tratamiento SL, generó pérdidas de $\mathrm{NO}_{3}{ }_{3}$, por lo que se genera acumulación y mayor toma de $\mathrm{N}_{-} \mathrm{NH}_{4}{ }^{+}$, ocasionado acidificación del sustrato. Bar-Yosef et al. (2009) demostraron que cambios en la relación $\mathrm{NO}_{3}{ }^{-} \mathrm{NH}_{4}{ }^{+}$de 75:25 a 50:50 impacta el $\mathrm{pH}$ de la rizosfera, de 8 a 4. Claussen \& Lenz (1999), en fresa con fertilización nítrica o amoniacal, concluyeron que el aumento en la oferta de $\mathrm{N}_{-} \mathrm{NH}_{4}{ }^{+}$disminuye la fotosíntesis neta, impactando la acumulación de masa seca. El exceso de agua genera pérdidas de $\mathrm{N}_{-} \mathrm{NO}_{3}{ }^{-}$, mayor proporción de $\mathrm{N}-\mathrm{NH}_{4}{ }^{+}$ disponible para las raíces, acidificación del medio, menor contenido de $\mathrm{N}$ en tejidos y menor acumulación de masa seca en la planta.

En la dinámica de $\mathrm{K}$, se constatan diferencias estadísticas en la acumulación del nutriente en tejido en las 21 y 28 SDS, con menor valor en el tratamiento SL (Figura 3c). Respuesta relacionada con el contenido de ${\mathrm{N}-\mathrm{NO}_{3}}_{3}$ en el sustrato, cambio en la relación $\mathrm{NO}_{3}{ }_{2} \mathrm{NH}_{4}{ }^{+}$y competencia directa de $\mathrm{NH}_{4}{ }^{+}-\mathrm{K}^{+}$, por ingreso a la planta; estos dos nutrientes comparten transportadores y canales de ingreso en membranas radicales, con prioridad para el $\mathrm{N}-\mathrm{NH}_{4}{ }^{+}$ (Ten Hoopen et al. 2010). Ten Hoopen et al. (2010), en arabidopsis y cebada, concluyeron que los flujos netos de $\mathrm{NH}_{4}^{+}$y $\mathrm{K}^{+}$se correlacionaron negativamente, al igual que sus concentraciones tisulares, lo que sugiere la existencia de competencia directa durante la absorción. Bar-Yosef et al. (2009) evaluaron las relaciones nitratoamonio-urea en rosa cultivada en sustrato, encontrando que la mejor relación $\mathrm{NO}_{3}{ }^{-} \mathrm{NH}_{4}{ }^{+}$para la absorción de $\mathrm{K}$ fue 88:12, a partir, de la cual, la acumulación de $\mathrm{K}$, en tejido, se afecta hasta en un 50 $\%$, en la relación $\mathrm{NO}_{3}: \mathrm{NH}^{+}$50:50, afectando los componentes de rendimiento en un $49 \%$. Según los datos para $\mathrm{K}$ en tejido, en sustrato y en lixiviado, una menor concentración en el tejido no estaría relacionada con pérdidas por lixiviación; por el contrario, el tratamiento SL muestra estadísticamente menor absorción del nutriente y mayor contenido en el sustrato, de lo cual, se infieren limitantes en absorción por antagonismos y no por pérdidas en el sistema.

Para el tratamiento SL, a partir de la 14 SDS, se observa una tendencia de disminución en la concentración de $\mathrm{Mg}$ en el tejido, con diferencia significativa en la 28 SDS (Figura 3d). Las dinámicas de $\mathrm{Mg}$ y $\mathrm{K}$ en sustrato y lixiviado son similares, con tendencia a mayores contenidos en el tratamiento SL, durante las 14, 21 y 28 SDS. En particular, la concentración de $\mathrm{Mg}$ en el sustrato es significativamente mayor en la 21 SDS, para el manejo SL, en comparación con el tratamiento CL (Figura 1e). Al considerar el argumento que el cambio en la relación $\mathrm{NO}_{3}: \mathrm{NH}_{4}{ }^{+}$generado por la lixiviación de la fuente nítrica y lo reportado por Lasa et al. (2000), quienes demostraron que el N-NH${ }_{4}^{+}$genera menores contenidos de $\mathrm{Mg}$ en plantas de girasol, cultivadas en un sistema hidropónico, es posible inferir un antagonismo similar al del K. En las plantas tratadas con $\mathrm{NH}_{4}{ }^{+}$, se afecta la cantidad de $\mathrm{Mg}$ en tejido, la masa seca, el área foliar, la fotosíntesis neta y la conductancia estomática, procesos que se restauran al tratar las plantas con dosis crecientes de $\mathrm{Mg}$, en la solución del fertirriego. Este impacto en los procesos mencionados está relacionado con la limitación generada sobre el proceso de carga de floema y traslocación de asimilados, por deficiencia de $\mathrm{Mg}$, lo que desencadena una sobreconcentración de azúcares en las hojas fuente, con la limitación de su transporte a otros órganos, impactando el proceso de crecimiento y de ganancia de masa seca en la planta (Fischer \& Bremer, 1993; Kakmak \& Kirkby, 2008).

En conclusión, las plantas de miniclavel cultivadas en sustrato con fertirriego monitoreado con lisímetro de pesada tendieron a acumular más masa seca que las plantas con el tratamiento con fertirriego tradicional. El uso del lisímetro en el manejo del fertirriego en esta especie frente al sistema tradicional generó un ahorro de 13,2 \% de agua y nutrientes con mayor eficiencia en absorción de $\mathrm{N}, \mathrm{K}$ y Mg. Además, por la concentración de nitrato encontrada en los lixiviados, se reafirma el potencial contaminante de las fórmulas de fertirriego, utilizadas en la producción de clavel.

Agradecimientos. A C.I. Florval, por el suministro del material vegetal de propagación y a Brenntag Colombia S.A., por la provisión de las soluciones fertilizantes utilizadas durante desarrollo de la investigación. Conflicto de intereses: El manuscrito fue preparado y revisado con la participación de todos los autores, quienes declaramos que no existe conflicto de intereses que ponga en riesgo la validez de los resultados presentados.

\section{REFERENCIAS}

1. ABASI, H.; BABALAR, M.; LESSANI, H.; NADERI, R. 2016. Effects of nitrogen form of nutrient solution on uptake and concentration macro element and morphological trait in hydroponic tulip. J. Plant Nutr. 39(12):1745-1751. https://doi.org/10.1080/01904167.2016.1201110

2. ARREAZA, P. 2000. Técnicas básicas del cultivo del clavel. En: Pizano de Márquez, M. (Ed.). Clavel (Dianthus caryophyllus). Ediciones Hortitecnia (Bogotá). p.15-40.

3. BARACALDO, A. DEL P.; DÍAZ, M.C.; FLÓREZ, V.J.; GONZÁLEZ, C.A. 2018. Efecto de la disminución de N total y aumento de $\mathrm{NH} 4+$ en la fórmula de fertirriego en el cultivo de clavel. Rev. Colomb. Cienc. Hortic. 12(3):658-667. https://doi.org/10.17584/rcch.2018v12i3.8062

4. BAR-YOSEF, B.; MATTSON, N.S.; LIETH, H.J. 2009. Effects of NH4:NO3:urea ratio on cut roses yield, leaf nutrients 
content and proton efflux by roots in closed hydroponic system. Sci. Hortic. (Holanda). 122(4):610-619.

https://doi.org/10.1016/j.scienta.2009.06.019

5. BARRERA-AGUILAR, E.; VALDEZ-AGUILAR, L.A.; CASTILLO-GONZÁLEZ, A.M.; IBARRA-JIMÉNEZ, L.; RODRÍGUEZ-GARCÍA, R.; ALIA-TEJACAL, I. 2012. La nutrición potásica afecta el crecimiento y fotosíntesis en Lilium cultivado en turba ácida. Rev. Mex. Cienc. Agríc. 3(5):1011-1022.

https://doi.org/10.29312/remexca.v3i5.1410

6. BERTSCH, F. 2003. Absorción de nutrimentos por los cultivos. Asociación Costarricense de la Ciencia del Suelo (Costa Rica). 307p.

7. BLOOM, A.J.; SUKRAPANNA, S.S.; WARNER, R.L. 1992. Root respiration associated with ammonium and nitrate absorption and assimilation by barley. Plant Physiol. (Estados Unidos). 99(4):1294-1301. https://doi.org/10.1104/pp.99.4.1294

8. CARPENTER, S.R.; CARACO, N.F.; CORRELL, D.L.; HOWARTH, R.W.; SHARPLEY, A.N.; SMITH, V.H. 1998. Nonpoint pollution of surface waters with phosphorus and nitrogen. Ecol. Appl. (Estados Unidos). 8(3):559-568. https://doi.org/10.2307/2641247

9. CASANOVA P., M.; MESSING, I.; JOEL, A.; CAÑETE M., A. 2009. Methods to estimate lettuce evapotranspiration in greenhouse conditions in the central zone of Chile. Chilean J. Agric. Res. 69(1):60-70.

https://doi.org/10.4067/S0718-58392009000100008

10. CLAUSSEN, W.; LENZ, F. 1999. Effect of ammonium or nitrate nutrition on net photosynthesis, growth, and activity of the enzymes nitrate reductase and glutamine synthetase in blueberry, raspberry and strawberry. Plant Soil. (Alemania). 208(5):95-102.

https://doi.org/10.1023/A:1004543128899

11. DAT, J.F.; CAPELLI, N.; FOLZER, H.; BOURGEADE, P.; BADOT, P-M. 2004. Sensing and signaling during plant flooding. Plant Physiol. Biochem. (Francia). 42(4):273-282. https://doi.org/10.1016/j.plaphy.2004.02.003

12. FAGERIA, N.K. 2009. The use of nutrients in crop plants. Taylor \& Francis Group (Boca Raton, FL). p.90-211.

13. FISCHER, E.S.; BREMER, E. 1993. Influence of magnesium deficiency on rates of leaf expansion, starch and sucrose accumulation, and net assimilation in Phaseolus vulgaris. Physiol. Plant. (Escandinavia). 89(2):271-276. https://doi.org/10.1111/j.1399-3054.1993.tb00153.x

14. FISHER, D.K. 2012. Simple weighing lysimeters for measuring evapotranspiration and developing crop coefficients. Int. J. Agric. \& Biol. Eng. (Estados Unidos). 5(3):35-43.

15. FLÓREZ R., V.J.; PARRA R., R.; RODRÍGUEZ S., M.; NIETO C., D.E. 2006. Características y fundamentos del proyecto "Producción más limpia de rosa y clavel con dos técnicas de cultivo sin suelo en la Sabana de Bogotá". En: Flórez R., V.J.; Fernández, A. de la C.; Miranda L., D.; Chaves C., B.; Guzman P., J.M. (eds). Avances sobre fertirriego en la floricultura colombiana. Unibiblios (Bogotá). p.3-40.

16. GONZÁLEZ-MURILLO, C.A.; LÓPEZ-ZULUAGA, A.; QUINTERO C., M.F.; FLÓREZ R., V.J. 2021. Evaluación funcional y aplicación de fertirriego en sustratos. En: Flórez R., V.J. (ed.). Productos relevantes del proyecto Fortalecimiento de la competitividad del sector floricultor colombiano mediante el uso de ciencia, tecnología e innovación aplicadas en Cundinamarca. Bogotá: Ediciones Unisalle, Ceniflores (Colombia). p.48-95.

17. GONZÁLEZ MURILLO, C.A.; QUINTERO BERTEL, Q.R.; FLÓREZ R., V.J.; QUINTERO C., M.F. 2017. Lisímetros volumétricos. En: Flórez R., V.J. (Ed). Consideraciones sobre producción, manejo y poscosecha de flores de corte con énfasis en rosa y clavel. Unibiblios (Bogotá). p.135-166.

18. GU, L.; LIU, P.; SHAO, L.; WANG, J.; DONG, S.; ZHAO, B.; SO, H.B.; SUN, W.; ZHANG, J.; ZHAO, B. 2014. A lisymeters study of Chinese wheat and maize varieties: I. The lysimeters-rain shelter facility and the growth and water use of wheat. Soil Tillage Res. (Holanda). 144(3):133-140. https://doi.org/10.1016/j.still.2014.06.005

19. HAWKESFORD, M.; HORST, W.; KICHEY, T.; LAMBERS, H.; SCHJOERRING, J.; MØLLER, I.S.; WHITE, P. 2012. Functions of macronutrients. En: Marschner, P. (ed.). Marschner's mineral nutrition of higher plants (3a. ed.). Academic Press (Amsterdam). p.135-189. https://doi.org/10.1016/B978-0-12-384905-2.00006-6

20. INSTITUTO DE HIDROLOGÍA, METEOROLOGÍA Y ESTUDIOS AMBIENTALES, IDEAM. 2021. Promedios Climatológicos 1981 - 2010. Disponible desde Internet en: http://www.ideam.gov.co/web/tiempo-y-clima/clima (con acceso el 06/08/2021).

21. KAFKAFI, U.; TARCHITZKY, J. 2011. Fertigation. A tool for efficient fertilizer and water management. International Fertilizer Industry Association e International Potash Institute (Francia). 141p.

22. KAKMAK, I.; KIRKBY, E.A. 2008. Role of magnesium in carbon partitioning and alleviating photooxidative damage. Physiol. Plant. (Escandinavia). 133(4):692-704. https://doi.org/10.1111/j.1399-3054.2007.01042.x 
23. KELSO B., H.-A.; BÂ, K.-M.; SÁNCHEZ M., S.; REYES L., D. 2012. Automatización de un lisímetro de pesada. Rev. Mex. Cienc. Agríc. 4:807-811.

24. KLEIBER, T.; KOMOSA, A. 2008. Comparison dynamics of $\mathrm{N}, \mathrm{P}, \mathrm{K}$ contents in different anthurium cultivars (Anthurium cultorum Birdsey) grown in expanded clay. Acta Sci. Pol., Hortorum Cultus (Polonia). 7(4):77-88.

25. LASA, B.; FRECHILLA, S.; ALEU, M.; GONZÁLEZ-MORO, B.; LAMSFUS, C.; APARICIO-TEJO, P.M. 2000. Effects of low and high levels of magnesium on the response of sunflower plants grown with ammonium and nitrate. Plant Soil. (Alemania). 225(1):167-174.

https://doi.org/10.1023/A:1026568329860

26. LI, S.; PEZESHKI, S.R.; GOODWIN, S. 2004. Effects of soil moisture regimes on photosynthesis and growth in cattail (Typha latifolia). Acta Oecol. (Holanda) 25(1):17-22. https://doi.org/10.1016/j.actao.2003.10.004

27. MEIJER, J.; BOT, G.P.A.; STANGHELLINI, C.; UDINK TEN CATE, A.J. 1985. Development and application of a sensitive, high precision weighing lysimeter for use in greenhouses. J. Agric. Eng. Res. (Holanda). 32(4):321-336. https://doi.org/10.1016/0021-8634(85)90097-6

28. NAVARRO, B.; NAVARRO, G. 2003. Química agrícola. (2ª ed.). Mundi-Prensa (Madrid). p.142-159.

29. PACHECO AVILA, J.; PAT CANUL, R.; CABRERA SANSORES, A. 2002. Análisis del ciclo del nitrógeno en el medio ambiente con relación al agua subterránea y su efecto en los seres vivos. Ingeniería. (México). 6(3):73-81.

30. RODRÍGUEZ, R.; PIRE, R. 2008. Evapotranspiración diaria del tomate determinada mediante un lisímetro de pesada. Agronomía Trop. (Venezuela). 58(1):73-76.

31. SONNEVELD, C.; VOOGT, W. 2009. Plant nutrition of greenhouse crops. Springer (Dordrecht). 431p.

32. TEN HOOPEN, F.; CUIN, T.A.; PEDAS, P.; HEGELUND, J.N.; SHABALA, S.; SCHJOERRING, J.K.; JAHN, T.P. 2010. Competition between uptake of ammonium and potassium in barley and Arabidopsis roots: molecular mechanisms and physiological consequences. J. Exp. Bot. (Inglaterra). 61(9):2303-2315.

https://doi.org/10.1093/jxb/erq057

33. VÉLEZ CARVAJAL, N.A.; FLÓREZ RONCANCIO, V.J.; FLÓREZ RIVERA, A.F. 2014. Comportamiento de variables químicas en un sistema de cultivo sin suelo para clavel en la Sabana de Bogotá. Rev. Fac. Nal. Agr. Medellín. (Colombia). 67(2):7281-7290.

34. VÉLEZ-CARVAJAL, N.A.; MELO-MARTÍNEZ, S.E.; FLÓREZ-RONCANCIO, V.J. 2014. Comportamiento de $\mathrm{Ca}, \mathrm{Mg}$ y $\mathrm{S}$ en un sistema de cultivo sin suelo para clavel. Rev. Chapingo Ser. Hortic. (México). 20(2):171-185. https://doi.org/10.5154/r.rchsh.2013.10.038 